\title{
49. DATA REPORT: TRACE METAL COMPOSITION OF PORE WATER FROM SITES 855 THROUGH 858, MIDDLE VALLEY, JUAN DE FUCA RIDGE ${ }^{1}$
}

\author{
C. Geoffrey Wheat ${ }^{2}$ and Michael J. Mott ${ }^{2}$
}

\section{INTRODUCTION}

Pore waters were collected from each of the four sites drilled during Ocean Drilling Program Leg 139 in Middle Valley, Juan de Fuca Ridge. Each site was drilled in a distinct physical and geological setting. Site 855 is located at the eastern boundary fault of the Middle Valley rift, where seawater enters basement either along the normal fault or through an outcrop. Site 856 is located on a massive sulfide deposit and a nearby uplifted sediment hill. Site 857 was positioned to drill into the hydrothermal fluid reservoir that feeds active hydrothermal vents $1.5 \mathrm{~km}$ to the north. Site 858 is located in an area of active hydrothermal venting where effluent temperatures reach $275^{\circ} \mathrm{C}$. Pore waters were obtained by squeezing whole-round and quarterround sections of sediment core using a titanium squeezer designed by Manheim and Sayles (1974) and by in-situ extraction using the water sampler temperature probe (WSTP) (Barnes, 1988). Samples also were obtained using a pressure core sampler and the Los Alamos titanium sampler. Detailed descriptions of sampling methods are given by the Shipboard Scientific Party (Davis, Mottl, Fisher, et al., 1992).

In this data report, we present analyses of pore waters from Leg 139 for $\mathrm{Li}, \mathrm{Rb}, \mathrm{B}, \mathrm{Sr}, \mathrm{Ba}, \mathrm{Mn}, \mathrm{Fe}, \mathrm{Ni}$, and $\mathrm{Zn}$ (Tables 1-4 and Figures $1-5)$. Only pore waters from whole-round sections are shown in Figures 4 and 5 because evaporation and water-rock reactions may have altered the pore-water chemistry of the quarter-round sections (Davis, Mottl, Fisher, et al., 1992). The chlorinity of each sample is also listed in the tables as it provides a measure of pore-water dilution with surface seawater/drilling fluid and, in the WSTP samples, distilled water. Some samples have higher chlorinity than expected. These high chlorinities probably resulted from evaporation.

\section{ANALYTICAL METHODS AND ARTIFACTS}

$\mathrm{Li}$ and $\mathrm{Rb}$ were determined by flame emission spectrometry using a standard addition method similar to that of Stoffyn-Egli (1982) on a five-fold dilution of the unacidified sample used for shipboard analyses. We measured $\mathrm{Li}$ at $671.0 \mathrm{~nm}$ and $\mathrm{Rb}$ at $779.4 \mathrm{~nm}$, using a $0.2-\mathrm{nm}$ slit. To account for spectral interferences we measured the background intensity $0.4 \mathrm{~nm}$ above and below the peak intensity. The average background intensity was then subtracted from the peak intensity.

Squeezing pore water from sediment can artificially increase the concentrations of $\mathrm{K}, \mathrm{Li}$, and $\mathrm{Rb}$ in pore water. Early diagenesis typically results in a decrease in the pore-water concentration of $\mathrm{Li}$ with depth, as is observed in the upper few meters of the sediment column at Site 855; however, pore waters from the uppermost meter of the sediment column from Site 855 have Li concentrations greater than that measured in bottom seawater. This elevated $\mathrm{Li}$ concentration cannot be supported by diagenesis in the upper meter of the sediment column based on flux calculations; it must result from ion-exchange reactions during sample handling. Similarly, $\mathrm{Rb}$ artifacts are evident in pore waters from the upper $10 \mathrm{~m}$ of the sediment column from Sites

\footnotetext{
'Mottl, M.J., Davis, E.E., Fisher, A.T., and Slack, J.F. (Eds.), 1994. Proc. ODP. Sci. Results, 139: College Station, TX (Ocean Drilling Program).

2 School of Ocean and Earth Science and Technology, University of Hawaii, 1000 Pope Road, Honolulu, HI 96822, U.S.A.
}

855,856 , and 857 . Diffusive and advective processes mask any squeezing artifacts that may be evident from Site 858 . Some of the observed scatter in the $\mathrm{Li}$ and $\mathrm{Rb}$ data is caused by the pattern of pore fluid circulation (Site 858 ) and by processing lithified sediments that yielded only several $\mathrm{ml}$ of pore water upon squeezing at the maximum allowable pressure for periods of up to 12 hours (Sites 857 and 858) (Davis, Mottl, Fisher, et al., 1992).

Some of the in-situ samples were contaminated with $\mathrm{Li}$ and $\mathrm{Rb}$. Concentrations of $\mathrm{Li}$ in samples from the WSTP 10-mL titanium sample coil agree with concentrations from squeezed samples. The only in-situ samples that are contaminated with $\mathrm{Li}$ are the overflow samples from the WSTP(designated BO in the tables). These samples have concentrations of $\mathrm{Li}$ up to 10 times greater than expected. Lubri-
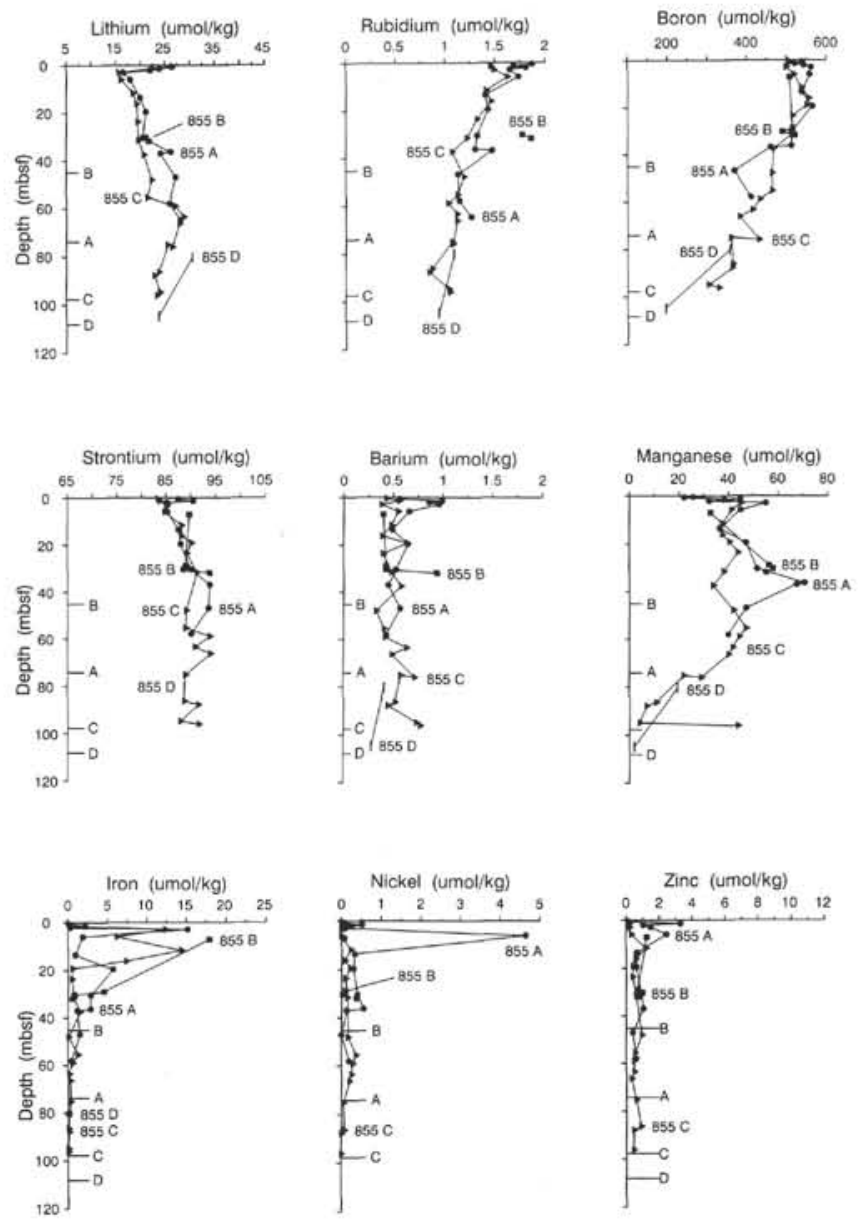

Figure 1. Trace metal composition of pore water in sediment from Site 855 . Hole $855 \mathrm{~A}=$ circles; Hole $855 \mathrm{~B}=$ squares; Hole $855 \mathrm{C}=$ triangles; and Hole $855 \mathrm{D}=$ bars. The depth at which basalt was encountered is labeled along the depth axis. 

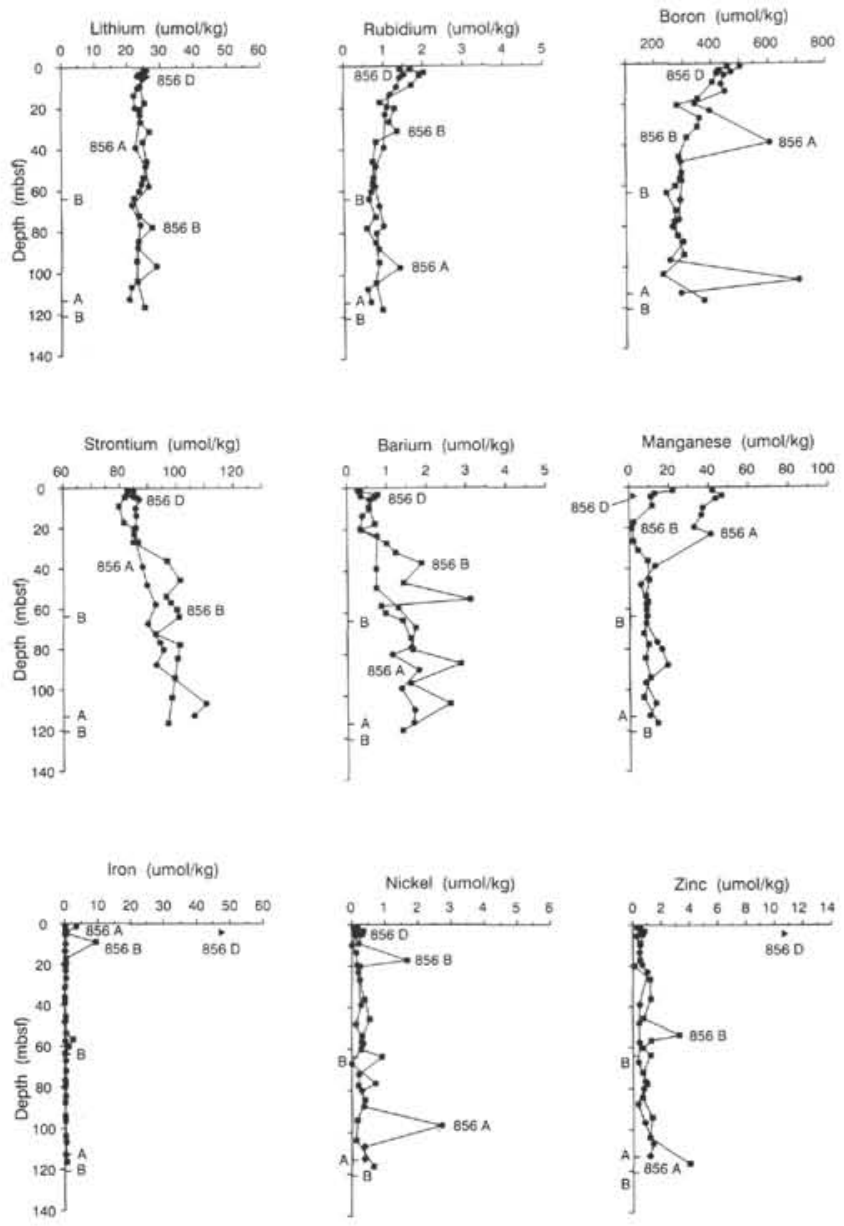

Figure 2. Trace metal composition of pore water in sediment from Site 856. Hole $856 \mathrm{~A}=$ circles; Hole $856 \mathrm{~B}=$ squares; and Hole $856 \mathrm{D}=$ triangles. The depth at which the tops of basaltic sills were encountered is labeled along the depth axis.

cating grease is the most likely source for this excess Li. Concentrations of $\mathrm{Rb}$ in samples from the WSTP $10-\mathrm{mL}$ titanium sample coil agree with concentrations from squeezed samples from Site 855, but are generally at least $50 \%$ greater than squeezed samples from the other three sites. A possible explanation is that during retrieval of sediment from Sites 856,857 , and 858 , all of which have temperature gradients greater than that at Site 855 , the concentration of dissolved $\mathrm{Rb}$ decreased by ion exchange caused by the change in temperature from in-situ values to room temperature. WSTP overflow samples (BO) generally have higher concentrations of $\mathrm{Rb}$ than samples from the 10-mL titanium sample coil.

$\mathrm{B}, \mathrm{Sr}, \mathrm{Ba}, \mathrm{Mn}, \mathrm{Fe}, \mathrm{Ni}$, and $\mathrm{Zn}$ were determined by inductively coupled plasma atomic emission spectrometry (ICPAES) on acidified samples. These samples were acidified with $0.015 \mathrm{~mL}$ of sub-boiled hydrochloric acid $(6 \mathrm{~N})$ per $10 \mathrm{~mL}$ of sample, and they were diluted fivefold before analysis. $\mathrm{Cu}$ also was determined but concentrations are not reported because samples were contaminated. Each site has concentrations of $\mathrm{Cu}$ that range from 0 to $13 \mu \mathrm{mol} / \mathrm{kg}$ with the exception of three samples with higher concentrations. Concentrations of $\mathrm{Cu}$ are highly scattered with depth, making trends difficult to visualize and impossible to interpret.

Concentrations of B are listed in Tables 1 to 4 . The value for surface seawater is $17 \%$ greater than it should be based on Millero's (1974) ratio of boron to chlorinity and the chlorinity of the surface seawater sample. For example, the surface seawater has a chlorinity of $497.6 \mathrm{mmol} / \mathrm{kg}$; the concentration of B should therefore be about
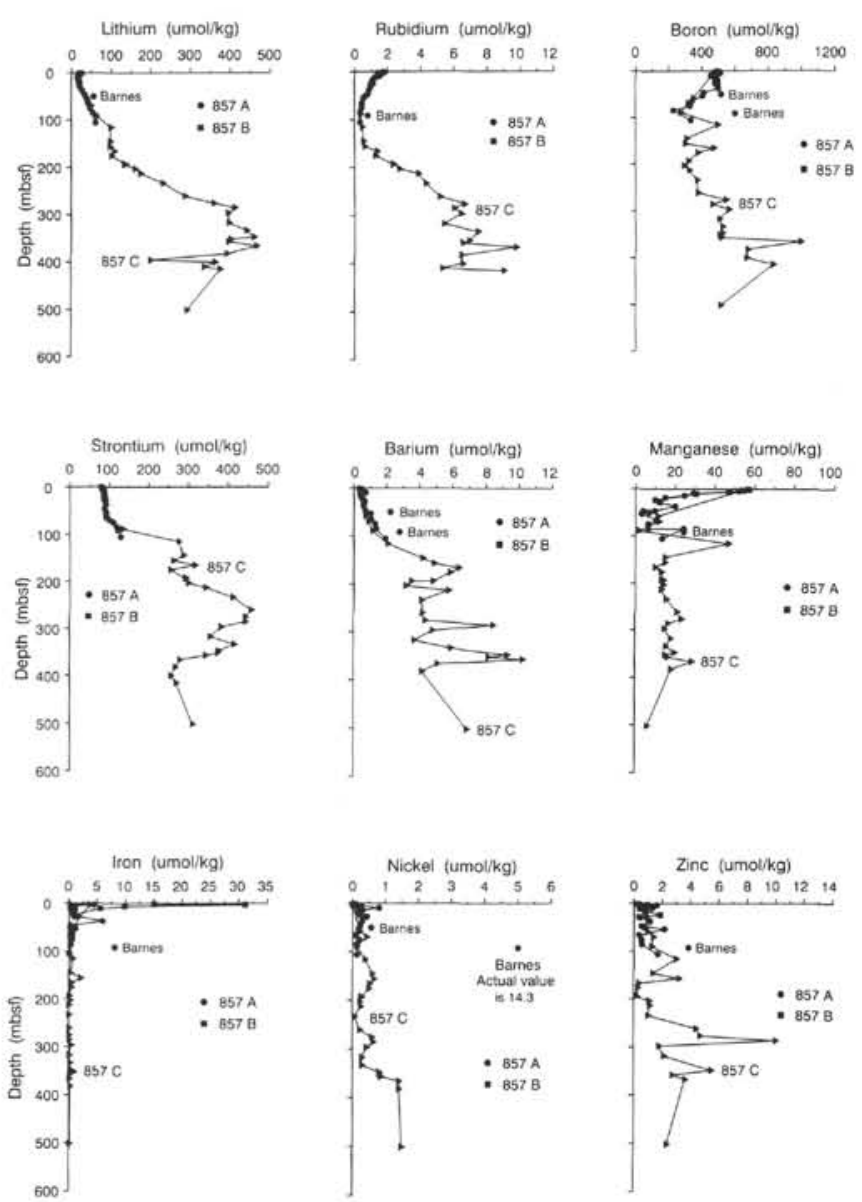

Figure 3. Trace metal composition of pore water in sediment and one sample of diabase (139-857C-62R-1, 142-150) from Site 857. Hole 857A = circles; Hole $857 \mathrm{~B}=$ squares; and Hole $857 \mathrm{C}=$ triangles. The Barnes label on the plots indicates analyses of the aliquot from the WSTP titanium sample coil.

$377 \mu \mathrm{mol} / \mathrm{kg}$, but the measured concentration is $440 \mu \mathrm{mol} / \mathrm{kg}$. Concentrations of B from Leg 139 pore waters also may be too high, but results obtained by analyzing samples with a range of B concentrations using the method of Grinstead and Snider (1967) agree with our results (Gieskes, pers. comm., 1993).

Differences in B concentration between in-situ samples and neighboring squeezed samples are insignificant for 11 of 15 samples. Two of the in-situ samples are about $20 \%$ lower than expected because of inclusion of bottom seawater in the sample coil; the remaining two samples are about $15 \%$ and $300 \%$ greater than expected. The sample with the greatest difference is $857 \mathrm{~A}-12 \mathrm{I}-1,74-97 \mathrm{~cm}$, which has an anomalously high chlorinity, possibly because the small $(5-\mathrm{mL})$ sample partially evaporated before analysis. This sample is also greatly contaminated with transition metals.

Concentrations of $\mathrm{Sr}$ and chlorinity in the surface seawater samples agree with Sr/chlorinity ratios presented by Millero (1974). In general, in-situ samples agree well with neighboring squeezed samples except for the cases where bottom seawater is entrained in the sample coil.

Concentrations of $\mathrm{Ba}, \mathrm{Mn}, \mathrm{Fe}, \mathrm{Ni}$, and $\mathrm{Zn}$ in pore waters from $\mathrm{Leg}$ 139 generally are greater than concentrations in the overlying bottom seawater. Concentrations of $\mathrm{Ba}$ and $\mathrm{Mn}$ are easily determined by ICPAES, in contrast to concentrations of $\mathrm{Fe}$ and $\mathrm{Ni}$ that are often at the limit of detection by ICPAES. Concentrations of $\mathrm{Zn}$ are low but detectable. The observed scatter in the data probably results from biological processes (e.g., Fe at Site 855), contamination (e.g., Ni at Site 
Table 1. Trace metal composition of pore water in sediment from Site 855.

\begin{tabular}{|c|c|c|c|c|c|c|c|c|c|c|c|c|}
\hline $\begin{array}{c}\text { Sample } \\
\text { type } / \text { /number }\end{array}$ & $\begin{array}{l}\text { Hole, core, section, } \\
\text { interval }(\mathrm{cm})\end{array}$ & $\begin{array}{l}\text { Depth } \\
\text { (mbsf) }\end{array}$ & $\begin{array}{l}\text { Chlorinity } \\
(\mathrm{mmol} / \mathrm{kg})\end{array}$ & $\begin{array}{c}\mathrm{Fe} \\
(\mu \mathrm{mol} / \mathrm{kg})\end{array}$ & $\begin{array}{c}\mathrm{Mn} \\
(\mu \mathrm{mol} / \mathrm{kg})\end{array}$ & $\begin{array}{c}\mathrm{Ni} \\
(\mu \mathrm{mol} / \mathrm{kg})\end{array}$ & $\begin{array}{c}\mathrm{Zn} \\
(\mu \mathrm{mol} / \mathrm{kg})\end{array}$ & $\begin{array}{c}\mathrm{Sr} \\
(\mu \mathrm{mol} / \mathrm{kg})\end{array}$ & $\begin{array}{c}\mathrm{Ba} \\
(\mu \mathrm{mol} / \mathrm{kg})\end{array}$ & $\begin{array}{c}\text { B } \\
(\mu \mathrm{mol} / \mathrm{kg})\end{array}$ & $\begin{array}{c}\mathrm{Li} \\
(\mu \mathrm{mol} / \mathrm{kg})\end{array}$ & $\begin{array}{c}\mathrm{Rb} \\
(\mu \mathrm{mol} / \mathrm{kg})\end{array}$ \\
\hline \multicolumn{3}{|c|}{ Surface seawater, 9 August 1991} & 497.6 & 0.05 & 0.00 & 0.02 & 0.01 & 83.1 & 0.02 & 440 & 23.3 & 1.28 \\
\hline IW-1 & $855 \mathrm{~A}-1 \mathrm{R}-1,55-70$ & 0.63 & 543.6 & 0.12 & 22.0 & 0.07 & 0.15 & 87.5 & 0.56 & 539 & 26.5 & 1.87 \\
\hline IW-2 & $855 \mathrm{~A}-1 \mathrm{R}-1,144-150$ & 1.47 & 539.1 & 2.17 & 44.8 & 0.52 & 3.30 & 90.5 & 0.99 & 521 & 23.9 & 1.69 \\
\hline IW-3 & $855 \mathrm{~A}-1 \mathrm{R}-2,53-67$ & 2.10 & 541.6 & 0.31 & 32.1 & 0.35 & 1.04 & 85.2 & 0.86 & 544 & 21.9 & 1.81 \\
\hline IW-4 & $855 \mathrm{~A}-1 \mathrm{R}-2,144-150$ & 2.97 & 537.7 & 15.2 & 55.1 & 0.43 & 1.49 & 85.4 & 0.96 & 562 & 16.5 & 1.65 \\
\hline IW-5 & $855 \mathrm{~A}-1 \mathrm{R}-4,144-150$ & 5.97 & 542.6 & 1.90 & 44.9 & 0.42 & 2.45 & 85.3 & 0.66 & 557 & 17.9 & 1.73 \\
\hline IW-6 & $855 \mathrm{~A}-2 \mathrm{R}-4,140-150$ & 13.55 & 543.6 & 0.89 & 36.4 & 0.33 & 0.63 & 87.6 & 0.49 & 538 & 19.9 & 1.40 \\
\hline IW-7 & $855 \mathrm{~A}-3 \mathrm{R}-2,140-150$ & 19.55 & 548.4 & 5.68 & 47.2 & 0.30 & 0.61 & 88.0 & 0.65 & 564 & 21.1 & 1.43 \\
\hline IW-8 & $855 \mathrm{~A}-4 \mathrm{R}-3,140-150$ & 30.45 & 551.4 & 2.86 & 51.7 & 0.40 & 0.91 & 90.1 & 0.53 & 513 & 20.5 & 1.32 \\
\hline BC-9 & $855 \mathrm{~A}-5 \mathrm{I}-1,74-97$ & 36.35 & 548.4 & 77.0 & 70.5 & 0.56 & 42.8 & 99.7 & 1.90 & 512 & 26.2 & 1.30 \\
\hline BO- $9^{b}$ & $855 \mathrm{~A}-5 \mathrm{I}-1,74-97$ & 36.35 & 382.4 & 1.25 & 66.2 & 73.6 & 24.4 & 95.6 & 1.34 & 456 & 47.8 & 1.28 \\
\hline IW-10 & $855 \mathrm{~A}-5 \mathrm{R}-1,140-150$ & 36.95 & 548.4 & 1.15 & 67.5 & 0.13 & 1.04 & 93.8 & 0.45 & 460 & 24.1 & 1.47 \\
\hline IW-11 & $855 \mathrm{~A}-6 \mathrm{R}-1,140-150$ & 46.95 & 544.5 & 1.48 & 47.2 & 0.00 & 0.40 & 93.6 & 0.57 & 369 & 27.2 & 1.13 \\
\hline IW-12 & $855 \mathrm{~A}-7 \mathrm{R}-2,140-150$ & 58.05 & 540.7 & 0.47 & 40.0 & 0.20 & 0.61 & 90.1 & 0.43 & 411 & 25.9 & 1.14 \\
\hline IW-13 & $855 \mathrm{~A}-8 \mathrm{R}-1,10-12$ & 64.91 & 537.7 & & & & & & & & 28.1 & 1.26 \\
\hline \multicolumn{2}{|c|}{ Depth to basalt: } & 74 & & & & & & & & & & \\
\hline IW-1 & $855 \mathrm{~B}-2 \mathrm{R}-1,140-150$ & 7.15 & 547.5 & 17.9 & 32.7 & 0.07 & 1.25 & 89.7 & 0.40 & 507 & & \\
\hline IW-2 & $855 \mathrm{~B}-4 \mathrm{R}-3,140-150$ & 28.95 & 546.5 & 4.52 & 56.5 & 0.10 & 0.67 & 89.0 & 0.43 & 515 & & \\
\hline IW-3 & $855 \mathrm{~B}-4 \mathrm{R}-4,130-144$ & 30.37 & 547.9 & 0.79 & 58.2 & 0.03 & 0.97 & 88.6 & 0.43 & 489 & 21.1 & 1.77 \\
\hline IW-4 & $855 \mathrm{~B}-4 \mathrm{R}-5,132-147$ & 31.90 & 559.1 & 0.52 & 55.2 & 0.38 & 0.82 & 93.8 & 0.94 & 521 & 21.6 & 1.86 \\
\hline \multicolumn{2}{|c|}{ Depth to basalt: } & 45 & & & & & & & & & & \\
\hline IW-1 & $855 C-1 R-1,40-52$ & 0.46 & 537.8 & 0.12 & 25.9 & 0.00 & 0.10 & 83.4 & 0.43 & 507 & 26.4 & \\
\hline IW-2 & $855 C-1 R-1,144-150$ & 1.47 & 538.7 & 0.23 & 38.4 & 0.10 & 0.19 & 83.7 & 0.56 & 504 & 22.5 & 1.46 \\
\hline IW-3 & $855 C-1 R-2,144-150$ & 2.97 & 542.6 & 12.4 & 45.1 & 0.00 & 0.22 & 85.1 & 0.38 & 500 & 15.6 & 1.49 \\
\hline IW-4 & $855 \mathrm{C}-1 \mathrm{R}-4,144-150$ & 5.97 & 541.6 & 6.20 & 41.2 & 0.00 & 0.33 & 84.7 & 0.55 & 518 & 16.0 & 1.62 \\
\hline IW-5 & $855 \mathrm{C}-2 \mathrm{R}-2,140-150$ & 11.65 & 541.6 & 14.3 & 37.5 & 0.23 & 1.19 & 88.2 & 0.48 & 537 & 18.5 & 1.41 \\
\hline IW-6 & $855 \mathrm{C}-2 \mathrm{R}-5,140-150$ & 16.15 & 547.5 & 7.34 & 37.5 & 0.08 & 0.57 & 88.2 & 0.39 & 555 & 19.2 & 1.46 \\
\hline IW-7 & $855 \mathrm{C}-3 \mathrm{R}-1,140-150$ & 19.15 & 550.4 & 0.60 & 40.5 & 0.22 & 0.40 & 90.2 & 0.64 & 550 & & \\
\hline IW-8 & $855 \mathrm{C}-3 \mathrm{R}-4,140-150$ & 23.65 & 551.3 & 0.52 & 44.0 & 0.10 & 0.39 & 89.3 & 0.40 & 515 & 19.5 & 1.32 \\
\hline IW-9 & $855 \mathrm{C}-4 \mathrm{R}-3,140-150$ & 31.55 & 554.2 & 0.75 & 38.2 & 0.15 & 0.60 & 91.2 & 0.48 & 513 & 19.6 & 1.22 \\
\hline BC-10 & $855 C-51-1,74-97$ & 37.45 & 544.5 & 1.52 & 33.7 & & & 80.3 & 0.58 & 468 & 20.7 & 1.07 \\
\hline $\mathrm{BO}-10^{\mathrm{b}}$ & $855 C-51-1,74-97$ & 37.45 & 185.7 & 0.00 & 21.9 & 8.82 & 9.28 & 91.1 & 1.21 & 402 & 177 & 1.38 \\
\hline IW-11 & $855 \mathrm{C}-6 \mathrm{R}-1,140-150$ & 47.95 & 535.8 & 0.10 & 42.1 & 0.17 & 0.96 & 89.1 & 0.33 & 464 & 22.3 & 1.19 \\
\hline IW-12 & $855 \mathrm{C}-6 \mathrm{R}-6,140-150$ & 55.45 & 537.8 & 1.29 & 47.2 & 0.38 & 0.55 & 89.0 & 0.41 & 465 & 21.4 & 1.13 \\
\hline IW-13 & $855 \mathrm{C}-7 \mathrm{R}-2,140-150$ & 59.05 & 547.5 & 0.61 & 44.4 & 0.30 & 0.48 & 93.9 & 0.42 & 433 & 27.0 & 1.03 \\
\hline IW-14 & $855 \mathrm{C}-7 \mathrm{R}-5,140-150$ & 63.55 & 544.5 & 0.12 & 41.9 & 0.25 & 0.51 & 90.9 & 0.64 & 415 & 28.8 & 1.12 \\
\hline IW-15 & $855 \mathrm{C}-8 \mathrm{R}-1,70-75$ & 66.43 & 544.5 & 0.26 & 40.1 & 0.20 & 0.31 & 94.0 & 0.49 & 382 & 27.9 & 1.12 \\
\hline $\mathrm{BC}-16$ & $855 \mathrm{C}-9 \mathrm{I}-1,74-97$ & 76.05 & 542.6 & 10.6 & 29.1 & & & 84.0 & 0.72 & 431 & 26.5 & 1.08 \\
\hline $\mathrm{BO}-16^{b}$ & $855 C-91-1,74-97$ & 76.05 & 477.8 & 0.14 & 32.9 & 2.55 & 10.51 & 88.4 & 0.64 & 482 & 42.0 & 1.37 \\
\hline IW-17 & $855 \mathrm{C}-9 \mathrm{R}-1,0-5$ & 75.23 & 532.9 & 0.35 & 22.0 & 0.05 & 0.60 & 89.0 & 0.58 & 360 & 25.6 & 1.07 \\
\hline IW-18 & $855 \mathrm{C}-10 \mathrm{R}-2,0-10$ & 86.35 & 537.2 & 0.05 & 10.7 & 0.08 & 0.90 & 88.6 & 0.52 & 365 & 23.8 & 0.87 \\
\hline IW-19 & $855 \mathrm{C}-10 \mathrm{R}-3,140-150$ & 87.85 & 537.7 & 0.16 & 6.8 & 0.00 & 0.45 & 91.5 & 0.45 & 363 & 22.9 & 0.84 \\
\hline IW-20 & $855 \mathrm{C}-11 \mathrm{R}-1,20-33$ & 94.87 & 541.0 & 0.05 & 4.1 & & & 87.9 & 0.74 & 304 & 24.0 & 1.04 \\
\hline IW-21 & $855 \mathrm{C}-11 \mathrm{R}-2,0-10$ & 96.15 & 538.0 & 0.07 & 43.9 & 0.00 & 0.45 & 91.6 & 0.78 & 330 & 23.4 & 1.05 \\
\hline \multicolumn{2}{|c|}{ Depth to basalt: } & 97.5 & & & & & & & & & & \\
\hline BC-1 & 855D-11- $1,74-97$ & 80.35 & 513.4 & 0.10 & 18.3 & & & 83.9 & 0.38 & 336 & 30.5 & 1.03 \\
\hline $\mathrm{BO}-1^{\mathrm{b}}$ & 855D-1I- $1,74-97$ & 80.35 & 355.9 & 0.13 & 17.7 & 2.66 & 2.52 & 85.0 & 0.35 & 354 & 53.5 & 1.02 \\
\hline IW -2 & $855 \mathrm{D}-4 \mathrm{R}-1,45-50$ & 104.78 & 535.8 & & 1.9 & & & 60.0 & 0.28 & 195 & 23.8 & 0.94 \\
\hline \multicolumn{2}{|c|}{ Depth to basalt: } & 108 & & & & & & & & & & \\
\hline
\end{tabular}

"IW = squeezed interstitial water sample; $\mathrm{BC}=$ sample from the titanium coil of the WSTP; $\mathrm{BO}=$ overflow aliquot from the WSTP (diluted with distilled water).

${ }^{b}$ Concentrations measured in overflow aliquots have been adjusted to the chlorinity of the prime aliquot.

856), squeezing highly lithified sediments (e.g., $\mathrm{Ba}$ and $\mathrm{Zn}$ at Site 857 ), or the circulation of pore waters (e.g., $\mathrm{Ba}, \mathrm{Mn}, \mathrm{Fe}, \mathrm{Ni}$, and $\mathrm{Zn}$ concentrations in Holes $858 \mathrm{~B}$ and $858 \mathrm{D}$ are greatest in the depth intervals where lateral fluid flow occurs).

In-situ samples from Site 855 are contaminated with $\mathrm{Ba}, \mathrm{Fe}, \mathrm{Ni}$, and $\mathrm{Zn}$. Both the overflow aliquot and aliquot from the titanium sample coil are affected. These artifacts are probably caused by oxidation during production and storage of the tool, as these were the first samples taken with the WSTP during Leg 139, and none of the other WSTP samples from the titanium sample coil are contaminated with $\mathrm{Fe}$. The grease that is used to seal the pressure case is another possible source of contamination. WSTP samples from Site 856 do not appear to be contaminated with $\mathrm{Ba}, \mathrm{Fe}, \mathrm{Mn}, \mathrm{Ni}$, and $\mathrm{Zn}$. Both of the overflow samples from Site 857 are contaminated with $\mathrm{Ni}$ and $\mathrm{Zn}$, and one of the two samples taken from the titanium sample coil is contaminated with all five metals $(\mathrm{Ba}, \mathrm{Mn}, \mathrm{Fe}, \mathrm{Ni}$, and $\mathrm{Zn})$. Ni and $\mathrm{Zn}$ contamination is evident in each of the overflow samples from Site 858. Fe contamination is evident in each of the sediment samples from the pressure core sampler at Site 858 , and $\mathrm{Zn}$ contamination is often evident in aliquots of the overlying water from the pressure core sampler. The
Mn data provide no evidence of a squeezing artifact or contamination in the in-situ samples, with the exception of one sample.

\section{ACKNOWLEDGMENTS}

This research was supported by the Ocean Drilling Program and by a grant from the Joint Oceanographic Institutions U.S. Science Support Program. We would like to thank Jim McManus for reviewing this manuscript. This is contribution number 3164 from the School of Ocean and Earth Science and Technology of the University of Hawaii.

\section{REFERENCES}

Barnes, R.O., 1988. ODP in-situ fluid sampling and measurement: a new wireline tool. In Mascle, A., Moore, J.C., et al., Proc. ODP, Init. Repts., 110: College Station, TX (Ocean Drilling Program), 55-63.

\footnotetext{
- Abbreviations for names of organizations and publications in ODP reference lists follow the style given in Chemical Abstracts Service Source Index (published by American Chemical Society).
} 
Davis, E.E., Mottl, M.J., Fisher, A.T., et al., 1992. Proc. ODP, Init. Repts., 139: College Station, TX (Ocean Drilling Program).

Grinstead, R.R., and Snider, S., 1967. Modification of the curcumin method for low level boron determinations. Analyst, 92:532-533.

Manheim, F.T., and Sayles, F.L., 1974. Composition and origin of interstitial waters of marine sediments based on deep sea drill cores. In Goldberg, E.D. (Ed.), The Sea (Vol. 5): New York (Wiley Interscience), 527-568.

Millero, F.J., 1974. Seawater as a multicomponent electrolyte solution. In Goldberg, E.D. (Ed.), The Sea (Vol. 5): New York (Wiley), 3-80.
Stoffyn-Egli, P., 1982. Conservative behavior of dissolved lithium in estuarine waters. Estuarine Coastal Shelf Sci., 14:577-587.

Date of initial receipt: 15 March 1993

Date of acceptance: 9 August 1993

Ms 139SR-269

Table 2. Trace metal composition of pore water in sediment from Site 856 .

\begin{tabular}{|c|c|c|c|c|c|c|c|c|c|c|c|c|}
\hline $\begin{array}{c}\text { Sample } \\
\text { type } / \text { number }\end{array}$ & $\begin{array}{l}\text { Hole, core, section, } \\
\text { interval }(\mathrm{cm})\end{array}$ & $\begin{array}{l}\text { Depth } \\
\text { (mbsf) }\end{array}$ & $\begin{array}{l}\text { Chlorinity } \\
(\mathrm{mmol} / \mathrm{kg})\end{array}$ & $\begin{array}{c}\mathrm{Fe} \\
(\mu \mathrm{mol} / \mathrm{kg})\end{array}$ & $\begin{array}{c}\mathrm{Mn} \\
(\mu \mathrm{mol} / \mathrm{kg})\end{array}$ & $\begin{array}{c}\mathrm{Ni} \\
(\mu \mathrm{mol} / \mathrm{kg})\end{array}$ & $\begin{array}{c}\mathrm{Zn} \\
(\mu \mathrm{mol} / \mathrm{kg})\end{array}$ & $\begin{array}{c}\mathrm{Sr} \\
(\mu \mathrm{mol} / \mathrm{kg})\end{array}$ & $\begin{array}{c}\mathrm{Ba} \\
(\mu \mathrm{mol} / \mathrm{kg})\end{array}$ & $\begin{array}{c}\text { B } \\
(\mu \mathrm{mol} / \mathrm{kg})\end{array}$ & $\begin{array}{c}\mathrm{Li} \\
(\mu \mathrm{mol} / \mathrm{kg})\end{array}$ & $\begin{array}{c}\mathrm{Rb} \\
(\mu \mathrm{mol} / \mathrm{kg})\end{array}$ \\
\hline \multicolumn{3}{|c|}{ Surface seawater 9 August 1991} & 497.6 & 0.05 & 0.00 & 0.02 & 0.01 & 83.1 & 0.02 & 440 & 23.3 & 1.28 \\
\hline IW-1 & $856 \mathrm{~A}-1 \mathrm{H}-2,0-6$ & 1.53 & 540.6 & 3.48 & 42.1 & 0.05 & 0.31 & 85.1 & 0.28 & 503 & 25.9 & 1.68 \\
\hline IW-2 & $856 \mathrm{~A}-2 \mathrm{H}-1,140-150$ & 4.15 & 540.6 & 0.52 & 46.9 & 0.07 & 0.58 & 85.0 & 0.35 & 471 & 23.1 & 1.50 \\
\hline IW-3 & $856 \mathrm{~A}-2 \mathrm{H}-2,140-150$ & 5.65 & 542.6 & 0.24 & 43.7 & 0.13 & 0.27 & 87.0 & 0.57 & 446 & 24.7 & 1.41 \\
\hline IW-4 & $856 \mathrm{~A}-2 \mathrm{H}-5,140-150$ & 10.15 & 539.7 & 0.19 & 37.1 & 0.00 & 0.52 & 85.7 & 0.56 & 435 & 23.1 & 1.32 \\
\hline IW-5 & $856 \mathrm{~A}-3 \mathrm{H}-1,143-150$ & 13.67 & 538.7 & 0.00 & 36.4 & 0.13 & 0.48 & 86.0 & 0.38 & 449 & 22.2 & 1.17 \\
\hline IW-6 & $856 \mathrm{~A}-3 \mathrm{H}-5,143-150$ & 19.67 & 540.6 & 0.35 & 32.7 & 0.15 & 0.66 & 85.8 & 0.33 & 343 & 22.5 & 1.10 \\
\hline IW-7 & $856 \mathrm{~A}-4 \mathrm{H}-1,140-150$ & 23.15 & 541.6 & 0.17 & 41.2 & 0.18 & 1.03 & 85.2 & 0.75 & 397 & 24.0 & 1.05 \\
\hline IW- 8 & $856 \mathrm{~A}-5 \mathrm{H}-6,140-150$ & 39.03 & 538.7 & 0.05 & 12.9 & 0.28 & 0.49 & 88.1 & 0.72 & 607 & 22.6 & 1.01 \\
\hline IW-9 & $856 \mathrm{~A}-6 \mathrm{H}-5,140-150$ & 48.15 & 538.7 & 0.00 & 5.6 & 0.13 & 0.45 & 89.7 & 0.72 & 292 & 25.6 & 0.80 \\
\hline IW-10 & $856 \mathrm{~A}-7 \mathrm{H}-5,140-150$ & 57.65 & 541.6 & 0.10 & 8.4 & 0.35 & 0.46 & 92.6 & 1.28 & 296 & 26.6 & 0.78 \\
\hline IW-11 & $856 \mathrm{~A}-8 \mathrm{H}-5,140-150$ & 67.15 & 540.6 & 0.28 & 8.2 & 0.00 & 0.39 & 89.9 & 1.71 & 290 & 21.4 & 0.89 \\
\hline IW- 12 & $856 \mathrm{~A}-9 \mathrm{H}-5,140-150$ & 76.65 & 543.6 & 0.05 & 13.9 & 0.71 & 0.87 & 94.3 & 1.62 & 285 & 24.0 & 0.99 \\
\hline IW- 13 & $856 \mathrm{~A}-10 \mathrm{X}-1,140-150$ & 80.15 & 540.6 & 0.02 & 16.4 & 0.30 & 0.75 & 95.6 & 1.14 & 262 & & 0.81 \\
\hline IW-14 & $856 \mathrm{~A}-11 \mathrm{X}-1,140-150$ & 87.65 & 538.7 & 0.00 & 19.2 & 0.37 & 0.34 & 93.0 & 1.80 & 300 & 23.1 & 0.87 \\
\hline BC- 15 & $856 \mathrm{~A}-12 \mathrm{I}-1,74-97$ & 96.55 & 539.7 & 0.09 & 8.0 & 2.73 & 0.82 & 55.7 & 1.36 & 252 & 28.8 & 1.39 \\
\hline BO- $15^{b}$ & $856 \mathrm{~A}-12 \mathrm{I}-1,74-97$ & 96.55 & 331.6 & & & & & & & & 71.5 & 1.58 \\
\hline IW-16 & $856 \mathrm{~A}-13 \mathrm{X}-1,140-150$ & 106.75 & 538.7 & 0.31 & 13.1 & 0.38 & 1.40 & 110 & 1.69 & 707 & 21.3 & 0.59 \\
\hline IW-17 & $856 \mathrm{~A}-13 \mathrm{X}-5,140-150$ & 112.75 & 534.8 & 0.05 & 10.0 & 0.38 & 1.15 & 106 & 1.67 & 293 & 20.7 & 0.67 \\
\hline \multicolumn{2}{|c|}{ Depth to basalt: } & 113.2 & & & & & & & & & & \\
\hline IW-1 & $856 \mathrm{~B}-1 \mathrm{H}-1,144-150$ & 1.47 & 538.7 & 0.12 & 22,0 & 0.17 & 0.54 & 83.0 & 0.33 & 458 & 25.1 & 1.42 \\
\hline IW-2 & $856 \mathrm{~B}-2 \mathrm{H}-1,140-150$ & 3.25 & 542.6 & 0.14 & 12.9 & 0.35 & 0.84 & 82.8 & 0.77 & 430 & 23.8 & 2.01 \\
\hline IW- 3 & $856 \mathrm{~B}-2 \mathrm{H}-2,140-150$ & 4.75 & 538.7 & 0.07 & 10.9 & 0.32 & 0.70 & 82.0 & 0.69 & 420 & 24.9 & 1.90 \\
\hline IW-4 & $856 \mathrm{~B}-2 \mathrm{H}-5,140-150$ & 9.25 & 541.6 & 9.40 & 11.6 & 0.20 & 0.52 & 79.8 & 0.55 & 405 & 23.8 & 1.70 \\
\hline IW-5 & $856 \mathrm{~B}-3 \mathrm{H}-4,140-150$ & 17.25 & 538.7 & 0.24 & 2.2 & 1.68 & 0.51 & 81.6 & 0.70 & 355 & 25.4 & 0.92 \\
\hline IW-6 & $856 \mathrm{~B}-3 \mathrm{H}-6,140-150$ & 20.25 & 540.6 & 0.00 & 1.3 & 0.23 & 0.12 & 85.3 & 0.35 & 280 & 24.0 & 1.29 \\
\hline IW-7 & $856 \mathrm{~B}-4 \mathrm{H}-4,140-150$ & 26.75 & 541.6 & 0.26 & 2.0 & 0.23 & 1.19 & 86.6 & 0.99 & 360 & 24.1 & 1.15 \\
\hline BC- 8 & $856 \mathrm{~B}-5 \mathrm{I}-1,74-97$ & 31.15 & 516.3 & 0.07 & 4.3 & & & 84.9 & 1.21 & 352 & 26.8 & 1.35 \\
\hline IW-9 & $856 \mathrm{~B}-5 \mathrm{H}-4,140-150$ & 36.25 & 537.7 & 0.09 & 9.2 & 0.40 & 1.28 & 96.8 & 1.88 & 314 & 24.8 & 0.81 \\
\hline IW -10 & $856 \mathrm{~B}-6 \mathrm{H}-4,140-150$ & 45.75 & 540.6 & 0.28 & 10.0 & 0.55 & 0.76 & 101 & 1.41 & 285 & 26.0 & 0.73 \\
\hline IW-11 & $856 \mathrm{~B}-7 \mathrm{H}-3,140-150$ & 53.75 & 539.7 & 0.42 & 8.1 & 0.32 & 1.82 & 96.4 & 3.10 & 295 & 24.9 & 0.74 \\
\hline IW-12 & $856 \mathrm{~B}-7 \mathrm{H}-5,140-150$ & 56.75 & 540.6 & 2.45 & 9.2 & 0.30 & 1.28 & 98.0 & 0.85 & 290 & 24.4 & 0.72 \\
\hline IW-13 & $856 \mathrm{~B}-8 \mathrm{H}-1,140-150$ & 60.25 & 536.8 & 1.19 & 8.5 & 0.28 & 0.72 & 100 & 0.95 & 273 & 23.6 & 0.68 \\
\hline IW-14 & $856 \mathrm{~B}-9 \mathrm{X}-1,140-150$ & 63.75 & 537.7 & 0.02 & 8.7 & 0.91 & 1.24 & 101 & 1.39 & 241 & 22.1 & 0.63 \\
\hline IW-15 & $856 \mathrm{~B}-10 \mathrm{H}-1,140-150$ & 72.25 & 517.3 & 0.33 & 7.3 & 0.22 & 0.69 & 92.8 & 1.61 & 274 & 23.6 & 0.79 \\
\hline IW-16 & $856 \mathrm{~B}-11 \mathrm{X}-3,140-150$ & 77.75 & 535.8 & 0.17 & 9.7 & 0.20 & 0.99 & 101 & 1.66 & 270 & 27.5 & 0.57 \\
\hline IW-17 & $856 \mathrm{~B}-12 \mathrm{X}-2,140-150$ & 84.55 & 534.8 & 0.23 & 7.9 & 0.40 & 0.66 & 101 & 2.86 & 280 & 23.3 & 0.78 \\
\hline IW- 18 & $856 \mathrm{~B}-13 \mathrm{X}-2,140-150$ & 94.05 & 536.8 & 0.00 & 10.4 & 0.17 & 1.34 & 99.5 & 1.59 & 302 & 22.8 & 0.87 \\
\hline IW-19 & $856 \mathrm{~B}-14 \mathrm{X}-2,140-150$ & 103.75 & 534.8 & 0.10 & 7.0 & 0.13 & 1.16 & 98.5 & 2.59 & 229 & 23.1 & 0.80 \\
\hline IW- 20 & $856 \mathrm{~B}-15 \mathrm{X}-4,140-150$ & 116.35 & 536.8 & 0.42 & 13.9 & 0.66 & 3.98 & 97.1 & 1.38 & 375 & 25.2 & 0.96 \\
\hline \multirow{2}{*}{\multicolumn{2}{|c|}{$\begin{array}{l}\text { Thin sill at: } \\
\text { Depth to basalt: }\end{array}$}} & 63.8 & & & & & & & & & & \\
\hline & & 120.7 & & & & & & & & & & \\
\hline IW-1 & $856 \mathrm{D}-1 \mathrm{H}-3,140-150$ & 4.45 & 536.8 & 47.3 & 2.0 & 0.20 & 10.7 & 86.2 & 0.72 & 423 & 25,9 & 1.52 \\
\hline
\end{tabular}

${ }^{a} \mathrm{IW}=$ squeezed interstitial water sample; $\mathrm{BC}=$ sample from the titanium coil of the WSTP; $\mathrm{BO}=$ overflow aliquot from the WSTP tool (diluted with distilled water).

${ }^{b}$ Concentrations measured in overflow aliquots have been adjusted to the chlorinity of the prime aliquot. 
Table 3. Trace metal composition of pore water in sediment and one sample of metadiabase (139-857C-62R-1, 142-150 cm) from Site 857.

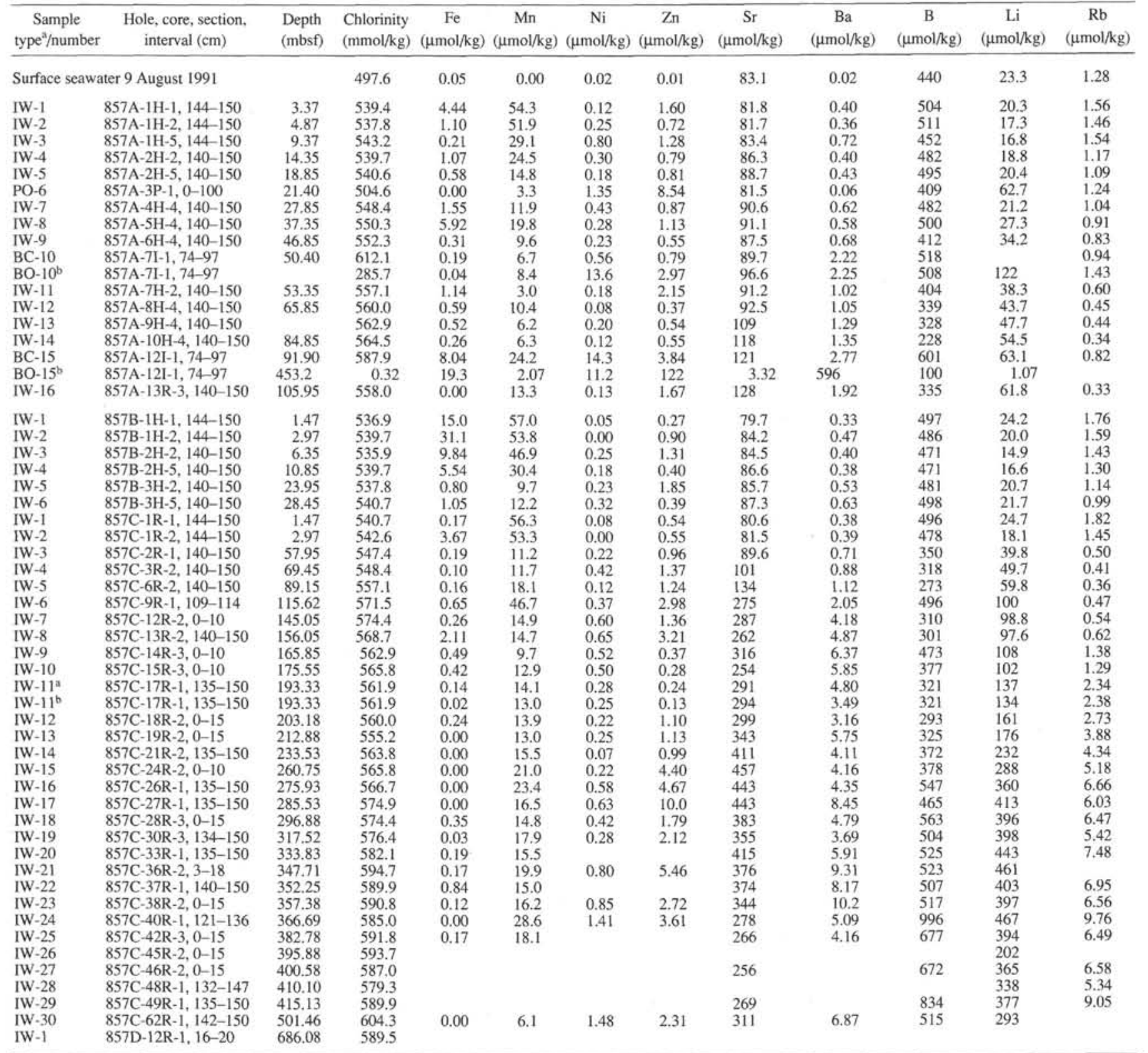

${ }^{\mathrm{a}} \mathrm{IW}=$ squeezed interstitial water sample; $\mathrm{BC}=$ sample from the titanium coil of the WSTP; $\mathrm{BO}=$ overflow aliquot from the WSTP (diluted with distilled water); PO = overflow aliquot from the pressure core sampler.

${ }^{b}$ Concentrations measured in overflow aliquots have been adjusted to the chlorinity of the prime aliquot. 
Table 4. Trace metal composition of pore water in sediment from Site 858.

\begin{tabular}{|c|c|c|c|c|c|c|c|c|c|c|c|c|}
\hline $\begin{array}{c}\text { Sample } \\
\text { type } / \text { /number }\end{array}$ & $\begin{array}{l}\text { Hole, core, section, } \\
\text { interval }(\mathrm{cm})\end{array}$ & $\begin{array}{l}\text { Depth } \\
\text { (mbsf) }\end{array}$ & $\begin{array}{l}\text { Chlorinity } \\
\text { (mmol/kg) }\end{array}$ & $\begin{array}{c}\mathrm{Fe} \\
(\mu \mathrm{mol} / \mathrm{kg})\end{array}$ & $\begin{array}{c}\mathrm{Mn} \\
(\mu \mathrm{mol} / \mathrm{kg})\end{array}$ & $\begin{array}{c}\mathrm{Ni} \\
(\mu \mathrm{mol} / \mathrm{kg})\end{array}$ & $\begin{array}{c}\mathrm{Zn} \\
(\mu \mathrm{mol} / \mathrm{kg})\end{array}$ & $\begin{array}{c}\mathrm{Sr} \\
(\mu \mathrm{mol} / \mathrm{kg})\end{array}$ & $\begin{array}{c}\mathrm{Ba} \\
(\mu \mathrm{mol} / \mathrm{kg})\end{array}$ & $\begin{array}{c}\text { B } \\
(\mu \mathrm{mol} / \mathrm{kg})\end{array}$ & $\begin{array}{c}\mathrm{Li} \\
(\mu \mathrm{mol} / \mathrm{kg})\end{array}$ & $\begin{array}{c}\mathrm{Rb} \\
(\mu \mathrm{mol} / \mathrm{kg})\end{array}$ \\
\hline \multicolumn{3}{|c|}{ Surface seawater 9 August 1991} & 497.6 & 0.05 & 0.00 & 0.02 & 0.01 & 83.1 & 0.02 & 440 & 23.3 & 1.28 \\
\hline IW-1 & $858 \mathrm{~A}-1 \mathrm{H}-1,144-150$ & 1.47 & 541.6 & 1.54 & 47.6 & 0.00 & 0.69 & 85.4 & 0.50 & 425 & 25.4 & 1.69 \\
\hline IW- 2 & $858 \mathrm{~A}-2 \mathrm{H}-2,144-150$ & 5.37 & 542.6 & 0.49 & 53.8 & 0.00 & 3.30 & 87.3 & 0.67 & 361 & 19.3 & 1.46 \\
\hline IW- -3 & $858 \mathrm{~A}-2 \mathrm{H}-3,144-150$ & 6.87 & 544.5 & 0.28 & 57.4 & 0.00 & 1.03 & 85.7 & 0.43 & 351 & 19.6 & 1.33 \\
\hline IW-4 & $858 \mathrm{~A}-2 \mathrm{H}-5,144-150$ & 9.87 & 541.0 & 0.02 & 46.5 & 0.00 & 0.52 & 87.7 & 0.72 & 360 & 22.4 & 1.46 \\
\hline IW-5 & $858 \mathrm{~A}-3 \mathrm{H}-4,140-150$ & 17.62 & 543.6 & 0.28 & 20.8 & 0.00 & 1.28 & 92.2 & 0.76 & 340 & 39.4 & 1.98 \\
\hline IW-6 & $858 \mathrm{~A}-4 \mathrm{H}-4,140-150$ & 27.35 & 545.5 & 0.09 & 21.3 & 0.00 & 1.01 & 96.4 & 1.06 & 373 & 62.2 & 2.97 \\
\hline IW-7 & $858 \mathrm{~A}-5 \mathrm{H}-4,140-150$ & 36.85 & 549.4 & 1.00 & 17.8 & 0.30 & 0.69 & 105 & 1.55 & 361 & 120 & 2.92 \\
\hline IW- 8 & $858 \mathrm{~A}-6 \mathrm{H}-2,140-150$ & 43.35 & 552.3 & 0.16 & 21.5 & 0.28 & 1.12 & 115 & 1.77 & 404 & 142 & 2.73 \\
\hline IW-9 & $858 \mathrm{~A}-7 \mathrm{H}-2,140-150$ & 52.85 & 554.2 & 1.01 & 22.7 & 0.28 & 0.66 & 140 & 2.42 & 410 & 165 & 2.11 \\
\hline IW-10 & $858 \mathrm{~A}-9 \mathrm{X}-2,0-10$ & 64.05 & 556.2 & 0.16 & 24.7 & 0.45 & 0.84 & 154 & 1.95 & 385 & 166 & 2.20 \\
\hline $1 W-11$ & $858 \mathrm{~A}-9 \mathrm{X}-5,140-150$ & 69.95 & 558.1 & 8.07 & 38.4 & 0.22 & 2.33 & 156 & 3.54 & 462 & 210 & 2.45 \\
\hline IW- 12 & $858 \mathrm{~A}-10 \mathrm{P}-1,0-8$ & 71.94 & 560.0 & 38.3 & 46.7 & 1.23 & 4.85 & 170 & 3.28 & 451 & 231 & 2.52 \\
\hline PO- 12 & $858 \mathrm{~A}-10 \mathrm{P}-1,0-8$ & 71.94 & 498.1 & 0.00 & 5.3 & 0.43 & 9.94 & 78.8 & 0.15 & 348 & 39.7 & 1.41 \\
\hline$B C-13$ & $858 \mathrm{~A}-111-1,74-97$ & 73.76 & 499.0 & 0.00 & 7.1 & 0.15 & 9.61 & 82.6 & 0.62 & 272 & 32.2 & 1.45 \\
\hline BO- $13^{b}$ & $858 \mathrm{~A}-111-1,74-97$ & 73.76 & 461.9 & 0.00 & 29.0 & 11.8 & 17,7 & 107 & 1.87 & 387 & 96.9 & 3.25 \\
\hline IW-14 & $858 \mathrm{~A}-11 \mathrm{X}-1,43-53$ & 73.38 & 555.2 & 0.00 & 36.4 & 0.27 & 7.46 & 171 & 2.90 & 440 & 213 & 2.51 \\
\hline IW- 15 & $858 \mathrm{~A}-15 \mathrm{X}-1,84-98$ & 111.51 & 566.9 & 0.42 & 35.7 & 0.28 & 3.79 & 216 & 3.14 & 525 & 275 & 3.82 \\
\hline IW-16 & $858 \mathrm{~A}-16 \mathrm{X}-1,52-62$ & 120.87 & 562.0 & 0.23 & 4.1 & 0.12 & 3.07 & 225 & 2.22 & 525 & 268 & 4.38 \\
\hline IW-17 & $858 \mathrm{~A}-18 \mathrm{X}-1,137-147$ & 141.02 & 544.0 & 0.35 & 42.3 & 0.18 & 1.43 & 146 & 2.15 & 356 & 182 & 3.92 \\
\hline IW- 18 & $858 \mathrm{~A}-20 \mathrm{X}-1,138-150$ & 160.37 & 549.4 & 0.37 & 32.7 & 0.12 & 1.96 & 171 & 1.93 & 297 & 188 & 2.08 \\
\hline IW-19 & $858 \mathrm{~A}-21 \mathrm{X}-2,90-100$ & 171.05 & 546.5 & 0.26 & 46.5 & 0.50 & 2.82 & 200 & 3.55 & 338 & 194 & 2.76 \\
\hline IW- -20 & $858 \mathrm{~A}-23 \mathrm{X}-1,22-31$ & 188.17 & 546.5 & 0.21 & 46.3 & 1.66 & 2.85 & 151 & 2.64 & 364 & 242 & 4.10 \\
\hline IW-21 & $858 \mathrm{~A}-25 \mathrm{X}-1,0-10$ & 207.35 & 538.7 & 0.07 & 92.5 & 0.23 & 2.36 & 136 & 2.43 & 379 & 251 & 5.61 \\
\hline IW- 22 & $858 \mathrm{~A}-27 \mathrm{X}-1,3-13$ & 226.68 & 544.5 & 0.28 & 49.7 & 0.91 & 4.15 & 156 & 4.62 & 452 & 270 & 6.08 \\
\hline IW- 23 & $858 \mathrm{~A}-29 \mathrm{X}-1,39-46$ & 246.33 & 575.5 & 0.56 & 20.1 & & & 227 & 2.37 & 1000 & 408 & 10.2 \\
\hline IW- 24 & $858 \mathrm{~A}-30 \mathrm{X}-1,90-102$ & 256.56 & 578.4 & 0.00 & 25.0 & 1.36 & 11.01 & 224 & 8.81 & 830 & 412 & 8.75 \\
\hline IW- -25 & $858 \mathrm{~A}-31 \mathrm{X}-1,69-84$ & 266.07 & 581.3 & & 6.3 & & & 251 & & 830 & 427 & 6.30 \\
\hline IW-26 & $858 \mathrm{~A}-32 \mathrm{X}-1,15-22$ & 274.69 & 578.4 & 0.16 & 10.8 & & & 234 & 2.93 & 901 & 443 & 8.41 \\
\hline IW- -27 & $858 \mathrm{~A}-34 \mathrm{X}-1,8-15$ & 291.62 & 556.5 & 0.09 & 20.1 & 0.85 & 9.27 & 230 & 2.47 & 617 & 325 & 7.11 \\
\hline IW-1 & $858 \mathrm{~B}-1 \mathrm{H}-1,140-150$ & 1.45 & 542.6 & 3.20 & 27.2 & 0.20 & 0.46 & 91.6 & 0.90 & 408 & 27.0 & 1.58 \\
\hline IW-2 & $858 \mathrm{~B}-1 \mathrm{H}-2,73-79$ & 2.26 & 563.5 & 0.00 & 25.2 & 0.38 & 1.70 & 95.0 & 1.14 & 299 & 31.6 & 1.62 \\
\hline IW-3 & $858 \mathrm{~B}-1 \mathrm{H}-2,140-150$ & 2.95 & 548.4 & 0.00 & 36.8 & 0.23 & 0.46 & 101 & 0.80 & 414 & 38.8 & 1.65 \\
\hline IW-4 & $858 \mathrm{~B}-1 \mathrm{H}-3,118-129$ & 4.24 & 573.2 & 0.00 & 11.7 & 0.28 & 0.25 & 115 & 0.79 & 183 & 51.2 & 2.39 \\
\hline IW-5 & $858 \mathrm{~B}-1 \mathrm{H}-4,38-44$ & 4.91 & 576.2 & 0.14 & 5.0 & 0.48 & 0.60 & 121 & 1.07 & 178 & 65.0 & 2.80 \\
\hline IW- 6 & $858 \mathrm{~B}-1 \mathrm{H}-4,140-150$ & 5.95 & 552.1 & 1.08 & 3.8 & 0.55 & 0.61 & 125 & 1.12 & 421 & 86.7 & 2.49 \\
\hline IW-7 & $858 \mathrm{~B}-2 \mathrm{H}-1,4 \mathrm{l}-73$ & 7.77 & 576.2 & 0.33 & 12.2 & 8.28 & 1.45 & 145 & 2.55 & 472 & 96.1 & 3.26 \\
\hline IW- 8 & $858 \mathrm{~B}-2 \mathrm{H}-2,7-21$ & 8.84 & 569.4 & 0.12 & 6.4 & 2.23 & 20.3 & 112 & 2.46 & 351 & 64.6 & 2.55 \\
\hline IW-9 & $858 \mathrm{~B}-2 \mathrm{H}-2,129-141$ & $\begin{array}{l}0.04 \\
10.05\end{array}$ & 572.3 & 0.05 & 6.5 & 1.01 & 4.94 & 137 & 3.71 & 515 & 140 & 4.45 \\
\hline IW- 10 & $858 \mathrm{~B}-2 \mathrm{H}-4,140-150$ & 13.15 & 589.4 & 26.2 & 21.3 & 1.16 & 0.07 & 252 & 11.0 & 1410 & 441 & 10.1 \\
\hline IW-11 & $858 \mathrm{~B}-2 \mathrm{H}-5,75-87$ & 14.01 & 606.3 & 0.05 & 45.1 & 4.65 & 6.22 & 288 & 7.53 & 1170 & 413 & 10.1 \\
\hline IW- 12 & $858 \mathrm{~B}-2 \mathrm{H}-6,17-32$ & 14.95 & 579.1 & 0.00 & 21.3 & 1.56 & 1.10 & 262 & 12.9 & 1070 & 309 & 11.5 \\
\hline IW- 13 & $858 \mathrm{~B}-2 \mathrm{H}-6,110-124$ & 15.87 & 578.1 & 0.07 & 10.9 & 0.71 & 0.40 & 256 & 19.2 & 966 & 314 & 10.5 \\
\hline IW- 14 & $858 \mathrm{~B}-3 \mathrm{H}-1,3-13$ & 16.78 & 557.4 & 10.6 & 74.4 & 0.15 & 0.15 & 234 & 4.90 & 1260 & 420 & 15.6 \\
\hline BC-15 & $858 \mathrm{~B}-41-1,74-97$ & 19.26 & 603.5 & & $1,4.4^{\circ}$ & & 0.12 & & & & 521 & 25.8 \\
\hline BO- $15^{\mathrm{b}}$ & $858 \mathrm{~B}-4 \mathrm{I}-1,74-97$ & 19.26 & 517.5 & 0.14 & 17.3 & 28.9 & 11.1 & 103 & 3.44 & 759 & 264 & 7.28 \\
\hline IW-16 & $858 \mathrm{~B}-5 \mathrm{H}-1,125-138$ & 25.22 & 622.9 & 0.09 & 12.8 & 0.66 & 9.55 & 326 & 7.09 & 911 & 489 & 14.4 \\
\hline IW-17 & $858 \mathrm{~B}-5 \mathrm{H}-2,143-150$ & 26.87 & 631.1 & & & & & & & & 589 & 14.0 \\
\hline IW- 18 & $858 \mathrm{~B}-5 \mathrm{H}-3,140-150$ & 28.35 & 616.6 & 14.6 & 11.0 & 0.88 & 0.10 & 353 & 95.2 & 1100 & 543 & 13.8 \\
\hline IW-1 & $858 \mathrm{C}-1 \mathrm{H}-1,144-150$ & 1.47 & 543.6 & 8.00 & 35.2 & 0.05 & 2.25 & 82.2 & 1.09 & 488 & 29.5 & 1.87 \\
\hline IW-2 & $858 \mathrm{C}-1 \mathrm{H}-2,144-150$ & 2.97 & 545.5 & 7.28 & 33.6 & 0.17 & 1.43 & 85.1 & 1.16 & 459 & 34.5 & 2.23 \\
\hline IW- 3 & $858 \mathrm{C}-2 \mathrm{H}-2,144-150$ & 6.47 & 546.5 & 0.00 & 45.3 & 0.12 & 0.58 & 85.4 & 1.59 & 379 & 42.5 & 2.55 \\
\hline IW- 4 & $858 \mathrm{C}-2 \mathrm{H}-3,140-150$ & 7.95 & 546.5 & 0.00 & 37.8 & 0.00 & 0.43 & 87.3 & 0.66 & 350 & 46.4 & 2.70 \\
\hline IW- 5 & $858 \mathrm{C}-2 \mathrm{H}-5,144-150$ & 10.97 & 549.4 & 7.84 & 33.4 & 0.18 & 3.21 & 93.5 & 0.96 & 305 & 53.2 & 2.39 \\
\hline IW- 6 & $858 \mathrm{C}-3 \mathrm{H}-3,144-150$ & 17.47 & 556.2 & 0.12 & 24.3 & 2.44 & 1.03 & 112 & 1.32 & 359 & 54.9 & 1.72 \\
\hline IW-7 & $858 \mathrm{C}-3 \mathrm{H}-5,144-150$ & 20.47 & 556.2 & 0.10 & 27.0 & 7.78 & 0.81 & 117 & 1.44 & 318 & 53.8 & \\
\hline BC- 8 & $858 \mathrm{C}-5 \mathrm{I}-1,74-97$ & 24.36 & 533.8 & 0.65 & 20.2 & 4.80 & 7.36 & 96.0 & 1.64 & 417 & 44.6 & 2.70 \\
\hline BO- $8^{b}$ & $858 \mathrm{C}-5 \mathrm{I}-1,74-97$ & 24.36 & 502.6 & 0.13 & 20.7 & 10.1 & 14.4 & 99.8 & 2.14 & 409 & 50.9 & 2.60 \\
\hline IW-9 & $858 \mathrm{C}-5 \mathrm{H}-4,140-150$ & 29.45 & 547.0 & 0.35 & 9.1 & 0.66 & 1.39 & 97.8 & 1.72 & 356 & 38.0 & 1.16 \\
\hline IW-10 & $858 \mathrm{C}-6 \mathrm{H}-3,140-150$ & 37.45 & 547.5 & 0.19 & 9.1 & 0.18 & 0.73 & 105 & 1.17 & 324 & 37.1 & 1.21 \\
\hline IW-11 & $858 \mathrm{C}-7 \mathrm{H}-1,140-150$ & 42.95 & 550.6 & 0.19 & 9.4 & 0.95 & 1.46 & 103 & 1.17 & 304 & 44.2 & 1.44 \\
\hline IW- 12 & $858 \mathrm{C}-10 \mathrm{aX}-\mathrm{CC}, 20-25$ & 48.03 & 546.5 & & & & & & & & 64.1 & 2.34 \\
\hline IW -12 & $858 \mathrm{C}-10 \mathrm{bX}-\mathrm{CC}, 20-25$ & 48.03 & 549.4 & 1.68 & 9.5 & 1.10 & 1.87 & 102 & 1.92 & 326 & 64.0 & 2.03 \\
\hline IW- 12 & $858 \mathrm{C}-10 \mathrm{X}-\mathrm{CC}, 30-35$ & $\begin{array}{l}40.03 \\
48.13\end{array}$ & 546.5 & 1.08 & 9.8 & & & & & & 97.9 & 2.64 \\
\hline IW- 13 & $858 \mathrm{C}-11 \mathrm{X}-1,5-15$ & 54.60 & 546.5 & 0.21 & 11.2 & 0.90 & 3.00 & 99.8 & 2.32 & 317 & 66.8 & 1.46 \\
\hline IW-14 & $858 \mathrm{C}-12 \mathrm{X}-1,131-145$ & 65.38 & 547.5 & 0.12 & 17.7 & 0.47 & 1.91 & 117 & 2.27 & 293 & 81.0 & 0.99 \\
\hline IW- 15 & $858 \mathrm{C}-13 \mathrm{X}-1,41-46$ & 74.14 & 545.5 & 0.30 & 43.0 & 0.48 & 5.31 & 155 & 4.20 & 412 & 103 & 3.08 \\
\hline IW-16 & $858 \mathrm{C}-14 \mathrm{X}-1,68-78$ & 84.13 & 556.2 & 15.4 & 28.9 & 0.00 & 0.19 & 145 & 12.4 & 529 & 103 & 3.11 \\
\hline IW-1 & $858 \mathrm{D}-1 \mathrm{H}-1,144-150$ & 1.47 & 596.1 & 0.16 & 5.1 & 0.05 & 0.03 & 183 & 2.95 & 886 & 150 & 7.19 \\
\hline IW-2 & $858 \mathrm{D}-1 \mathrm{H}-3,3-12$ & $\begin{array}{l}1.47 \\
3.08\end{array}$ & $\begin{array}{l}666.7 \\
66.7\end{array}$ & 0.03 & 17.6 & 0.43 & 2.22 & 257 & 49.8 & 758 & 281 & 11.4 \\
\hline IW- -3 & $858 \mathrm{D}-1 \mathrm{H}-3,144-150$ & 4.47 & 655.7 & 143 & 55.1 & 0.33 & 1.64 & 278 & 12.0 & 1220 & 336 & 10.1 \\
\hline IW-4 & $858 \mathrm{D}-1 \mathrm{H}-4,103-117$ & 5.60 & 675.4 & 0.00 & 15.2 & 0.63 & 1.6 & 310 & 116 & 614 & 352 & 11.9 \\
\hline IW- 5 & $858 \mathrm{D}-1 \mathrm{H}-5,144-150$ & 7.47 & 660.3 & 106 & 32.5 & 0.38 & 4.95 & 321 & 255 & 1340 & 422 & 9.30 \\
\hline IW- 6 & $858 \mathrm{D}-1 \mathrm{H}-6,128-142$ & 8.85 & 656.0 & 0.00 & 6.7 & 0.27 & 1.15 & 316 & 222 & 742 & 409 & 11.1 \\
\hline IW-7 & $858 \mathrm{D}-2 \mathrm{H}-2,2-16$ & 10.89 & 638.5 & 0.00 & 8.5 & 0.22 & 2.79 & 287 & 21.0 & 662 & 425 & 7.29 \\
\hline IW-8 & $858 \mathrm{D}-2 \mathrm{H}-2,120-133$ & 12.07 & 638.3 & 0.00 & 10.6 & 2.08 & 6.85 & 281 & 17.6 & 657 & 445 & 6.54 \\
\hline IW-9 & $-3,144-150$ & 13.77 & 59 & 5.90 & 15.0 & 0.70 & 0.19 & 271 & 16.0 & 1350 & 525 & 6.86 \\
\hline IW-10 & $858 \mathrm{D}-2 \mathrm{H}-4,133-147$ & 15.20 & 629.7 & 0.00 & 12.4 & 1.30 & 4.31 & 293 & 42.1 & 859 & 491 & 7.36 \\
\hline IW-11 & $858 \mathrm{D}-2 \mathrm{H}-5,144-150$ & 16.77 & 621.1 & 0.52 & 17.7 & 0.40 & 1.72 & 304 & 19.4 & 1060 & 474 & 4.46 \\
\hline IW- 12 & $858 \mathrm{D}-2 \mathrm{H}-6,133-147$ & 18.20 & 596.6 & 0.00 & 16.9 & 0.65 & 5.46 & 278 & 6.34 & 1160 & 473 & 8.20 \\
\hline IW- 13 & $858 \mathrm{D}-3 \mathrm{P}-1,0-13$ & 18.87 & 580.0 & 118 & 38.9 & 0.53 & 1.27 & 251 & 5.91 & 1310 & 468 & 7.23 \\
\hline PO- 13 & $858 \mathrm{D}-3 \mathrm{P}-1,0-13$ & 18.87 & 512.5 & 0.17 & 17.9 & 0.86 & 93.1 & 79.5 & 0.37 & 418 & 183 & 1.37 \\
\hline BC-14 & $858 \mathrm{D}-41-1,74-97$ & $\begin{array}{l}10.01 \\
20.66\end{array}$ & 569.8 & 34.4 & 31.8 & & & 240 & 36.9 & 1580 & 471 & 18.9 \\
\hline
\end{tabular}


Table 4 (continued).

\begin{tabular}{|c|c|c|c|c|c|c|c|c|c|c|c|c|}
\hline $\begin{array}{c}\text { Sample } \\
\text { type }{ }^{\mathrm{a}} / \text { number }\end{array}$ & $\begin{array}{l}\text { Hole, core, section, } \\
\text { interval }(\mathrm{cm})\end{array}$ & $\begin{array}{l}\text { Depth } \\
\text { (mbsf) }\end{array}$ & $\begin{array}{l}\text { Chlorinity } \\
(\mathrm{mmol} / \mathrm{kg})\end{array}$ & $\begin{array}{c}\mathrm{Fe} \\
(\mu \mathrm{mol} / \mathrm{kg})\end{array}$ & $\begin{array}{c}\mathrm{Mn} \\
(\mu \mathrm{mol} / \mathrm{kg})\end{array}$ & $\begin{array}{c}\mathrm{Ni} \\
(\mu \mathrm{mol} / \mathrm{kg})\end{array}$ & $\begin{array}{c}\mathrm{Zn} \\
(\mu \mathrm{mol} / \mathrm{kg})\end{array}$ & $\begin{array}{c}\mathrm{Sr} \\
(\mu \mathrm{mol} / \mathrm{kg})\end{array}$ & $\begin{array}{c}\mathrm{Ba} \\
(\mu \mathrm{mol} / \mathrm{kg})\end{array}$ & $\begin{array}{c}\text { B } \\
(\mu \mathrm{mol} / \mathrm{kg})\end{array}$ & $\begin{array}{c}\mathrm{Li} \\
(\mu \mathrm{mol} / \mathrm{kg})\end{array}$ & $\begin{array}{c}\mathrm{Rb} \\
(\mu \mathrm{mol} / \mathrm{kg}\end{array}$ \\
\hline BO- $14^{b}$ & 858D-4I-1, 74-97 & 20.66 & 532.9 & 63.5 & 36.3 & 15.3 & 4.28 & 250 & 11.2 & 1690 & 482 & 22.0 \\
\hline IW-15 & $858 \mathrm{D}-4 \mathrm{H}-2,56-80$ & 20.72 & 579.1 & 0.00 & 32.5 & 1.60 & 6.15 & 144 & 1.90 & 585 & 146 & 5.08 \\
\hline IW-16 & $858 \mathrm{D}-4 \mathrm{H}-2,144-150$ & 21.51 & 562.3 & 15.7 & 40.8 & 0.33 & 1.04 & 218 & 6.87 & 1270 & 368 & 5.89 \\
\hline IW-17 & $858 \mathrm{D}-4 \mathrm{H}-3,100-107$ & 22.58 & 600.5 & 0.00 & 9.8 & 2.73 & 2.91 & 253 & 12.7 & 1060 & 426 & 7.97 \\
\hline IW-18 & $858 \mathrm{D}-4 \mathrm{H}-4,104-118$ & 24.15 & 598.6 & 0.00 & 25.6 & 10.3 & 17.5 & 264 & 11.0 & 1070 & 455 & 6.15 \\
\hline IW-19 & $858 \mathrm{D}-4 \mathrm{H}-5,144-150$ & 26.01 & 585.4 & 0.24 & 20.8 & 2.06 & 0.46 & 275 & 42.6 & 1330 & 496 & 5.03 \\
\hline IW-20 & $858 \mathrm{D}-4 \mathrm{H}-6,134-149$ & 27.46 & 566.4 & 0.00 & 28.2 & 5.35 & 5.51 & 243 & 10.3 & 1090 & 410 & 7.38 \\
\hline IW-21 & $858 \mathrm{D}-6 \mathrm{X}-1,96-105$ & 38.21 & 569.1 & 3.23 & 29.3 & 0.60 & 0.19 & 225 & 3.62 & 1030 & 390 & 12.0 \\
\hline IW-1 & $858 \mathrm{~F}-9 \mathrm{R}-\mathrm{CC}, 0-1$ & 94.21 & 570.7 & & & & & & & & 422 & 17.5 \\
\hline IW-2 & $858 \mathrm{~F}-11 \mathrm{R}-1,0-3$ & 113.62 & 530.0 & 2.97 & 38.7 & & & 156 & 4.19 & 594 & 180 & 7.72 \\
\hline IW-3 & $858 \mathrm{~F}-14 \mathrm{R}-1,0-3$ & 142.62 & 570.7 & 13.3 & 27.5 & & & 246 & 3.91 & 1270 & 461 & 9.72 \\
\hline IW-4 & $858 \mathrm{~F}-17 \mathrm{R}-1,0-3$ & 171.62 & 561.5 & 33.4 & 11.7 & & & 224 & 14.4 & 1200 & 393 & 18.1 \\
\hline IW-5 & $858 \mathrm{~F}-18 \mathrm{R}-1,67-72$ & 182.00 & 567.8 & & & & & 221 & & 1070 & 389 & 16.5 \\
\hline IW- 6 & $858 \mathrm{~F}-19 \mathrm{R}-1,9-12$ & 191.01 & 565.8 & & & & & 212 & & 1070 & 333 & 15.1 \\
\hline IW-7 & $858 \mathrm{~F}-21 \mathrm{R}-1,9-13$ & 210.41 & 565.8 & 11.2 & 24.0 & & & 215 & 5.82 & 918 & 359 & 9.29 \\
\hline IW-8 & $858 \mathrm{~F}-24 \mathrm{R}-\mathrm{CC}, 0-4$ & 239.32 & 567.8 & 1.19 & 33.0 & & & 228 & 9.73 & 1240 & 448 & 20.4 \\
\hline IW-9 & $858 \mathrm{~F}-25 \mathrm{R}-1,2-8$ & 248.95 & 566.8 & 0.70 & 22.0 & 0.96 & 4.16 & 218 & 5.33 & 893 & 403 & 11.7 \\
\hline LA-1 & $858 \mathrm{~F}$ & & & 101.80 & 502.6 & 0.09 & 3.9 & 8.08 & 10.4 & 80.6 & 0.50 & 414 \\
\hline
\end{tabular}

${ }^{\mathrm{a}} \mathrm{IW}=$ squeezed interstitial water sample; $\mathrm{BC}=$ sample from the titanium coil of the WSTP; $\mathrm{BO}=$ overflow aliquot from the WSTP (diluted with distilled water); PO = overflow aliquot from the pressure core sampler; $L A=$ sample from the Los Alamos National Laboratory borehole water sampler.

${ }^{b}$ Concentrations measured in overflow aliquots have been adjusted to the chlorinity of the prime aliquot.
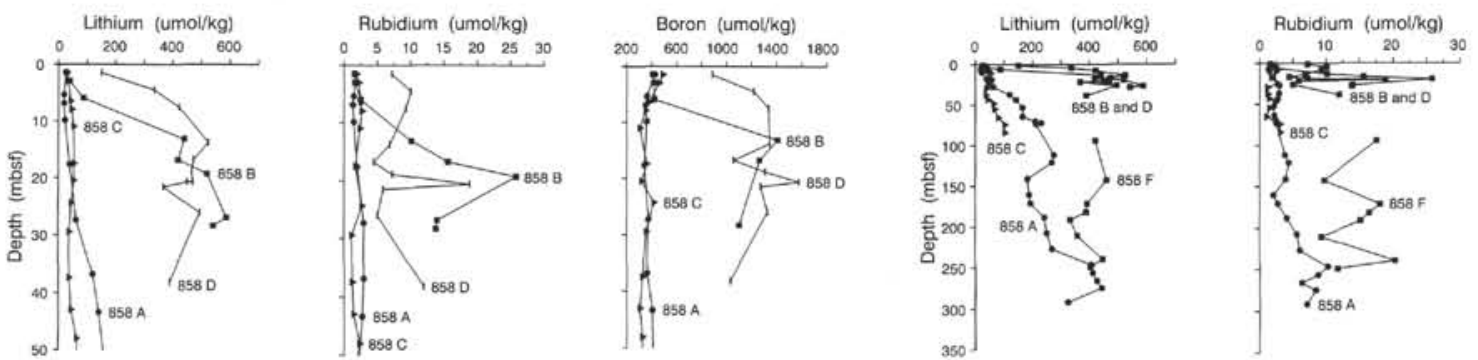

Boron (umol/kg)
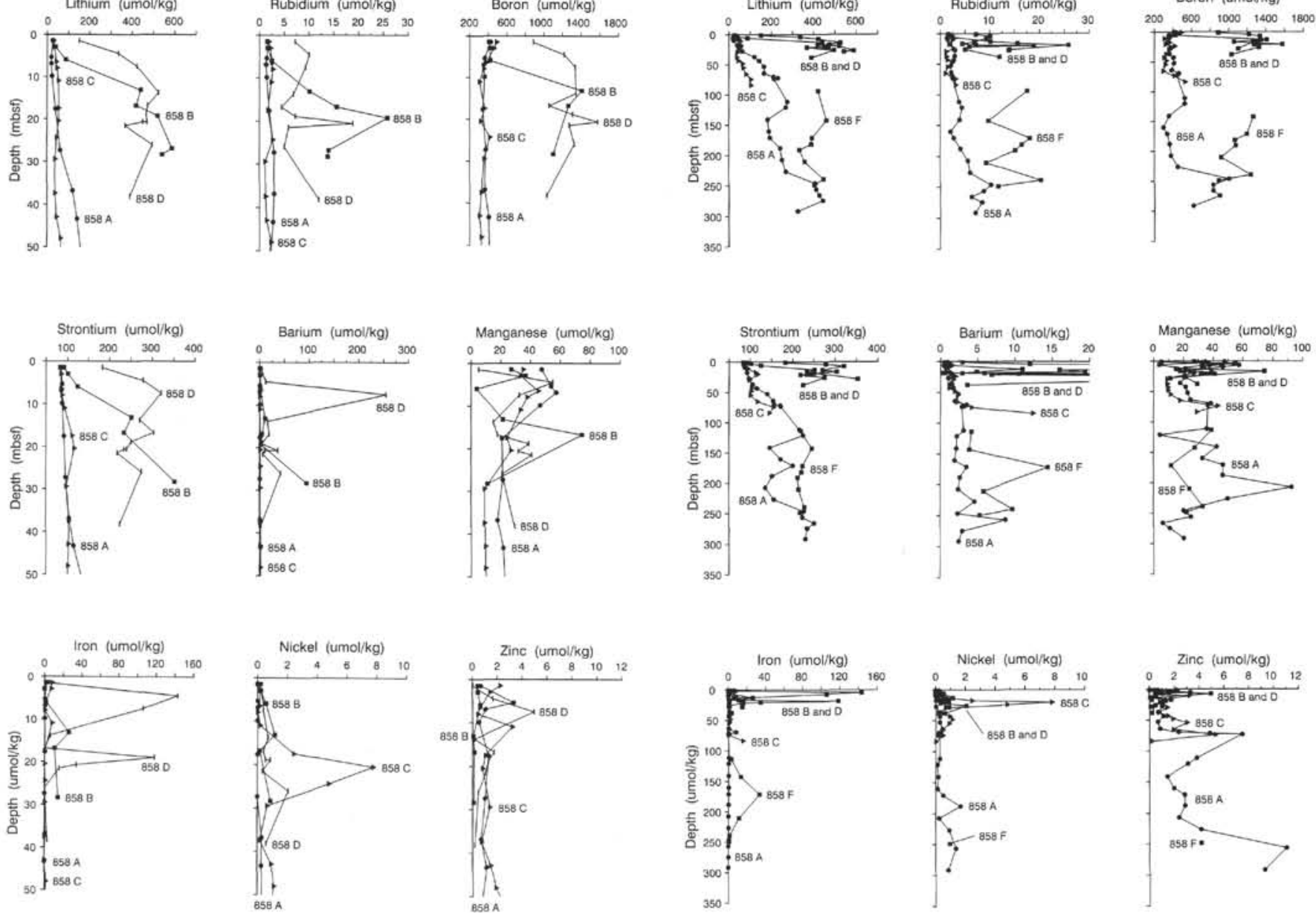

Figure 4. Trace metal composition of pore water in sediment from Site 858. Hole $858 \mathrm{~A}=$ circles; Hole $858 \mathrm{~B}=$ squares; Hole $858 \mathrm{C}=$ triangles; and Hole $858 \mathrm{D}=$ bars. Only the upper $50 \mathrm{mbsf}$ are shown and none of the quarter-round samples are plotted.

Figure 5. Trace metal composition of pore water in sediment from Site 858. Hole 858A = circles; Hole 858B = squares; Hole 858C = triangles; Hole 858D $=$ squares; and Hole $858 \mathrm{~F}=$ squares. None of the quarter-round samples are plotted. 